\title{
Il deficit di vitamina $D$ può rappresentare un fattore di rischio per la poliabortività attivando i meccanismi dell'immunità cellulare e dell'autoimmunità
}

\author{
Marco Centanni · Miriam Cellini
}

Pubblicato online: 5 giugno 2014

(c) Springer International Publishing AG 2014

\section{Commento a:}

Vitamin D deficiency may be a risk factor for recurrent pregnancy losses by increasing cellular immunity and autoimmunity.

K. Ota, S. Dambaeva, A.R. Han, K. Beaman,

A. Gilman-Sachs, J. Kwak-Kim.

Hum Reprod (2014) 29(2):208-219

L'attività biologica della vitamina $\mathrm{D}$, ormone steroideo implicato nell'omeostasi calcio-fosforica e nel metabolismo osseo, si espleta secondo due modalità: la risposta genomica lenta e la risposta non genomica rapida. È proprio attraverso quest'ultimo meccanismo che la vitamina D modula la risposta immunitaria innata e quella adattativa. Questi effetti immunoregolatori incidono anche sul sistema riproduttivo, soprattutto durante l'impianto della blastocisti. In particolare, nelle fasi iniziali della gravidanza, la vitamina $D$ viene prodotta dalle cellule del trofoblasto, agisce su di esse e condiziona il successo della gravidanza stessa, influenzando una risposta antinfiammatoria placentare e inducendo la decidualizzazione.

Il legame tra vitamina $\mathrm{D}$ e gravidanza è stato oggetto dello studio di Ota e collaboratori, che ha valutato la relazione tra i livelli sierici della vitamina e il pattern immunologico e citochinico Th1/Th2, nonché la citotossicità mediata dalle cellule NK nel sangue periferico di 133 donne poliabortive (3 o più aborti spontanei consecutivi entro la ventesima settimana di gestazione). Il $47 \%$ delle donne poliabortive presentava livelli insufficienti di vitamina D $(<30 \mathrm{ng} / \mathrm{ml})$ e la

M. Centanni $(\varangle) \cdot$ M. Cellini

Dipartimento di Scienze e Biotecnologie Medico-Chirurgiche, UOC di Endocrinologia, "Sapienza" Università di Roma, Latina,

Italia

e-mail: marco.centanni@uniroma1.it prevalenza di anticorpi antifosfolipidi, ANA, TPOAb e antissDNA era significativamente più alta nelle donne con inadeguati livelli di colecalciferolo. Inoltre, nel sangue periferico delle donne con ipovitaminosi sono risultati più elevati i livelli di linfociti B CD19+, segno indiretto dell'attivazione della risposta immunitaria umorale, nonché i livelli delle cellule NK CD56+ e della loro attività citotossica. Un dato ancora più interessante è emerso dallo studio in vitro dell' attività citotossica delle cellule NK e del rilascio di citochine Th1/Th2, dopo incubazione del campione di sangue periferico con 10 o $100 \mathrm{nM}$ di vitamina D. Quest'ultima era in grado, in maniera dose-dipendente, di sopprimere l'attività delle cellule NK, di ridurre la produzione di citochine Th1 (IFN- $\gamma$ e TNF- $\alpha$ ) e di incrementare il rilascio di citochine Th2 (IL-10, IL-1 $\beta$ ). Lo studio in vitro mostrava, inoltre, un aumento dei livelli di VEGF e G-CSF, fattori di crescita coinvolti nell' angiogenesi e nel trofismo placentare.

Da questi risultati emergono le molteplici implicazioni immunologiche che il deficit vitaminico può avere nelle donne con poliabortività. In particolare, la vitamina $\mathrm{D}$ è in grado di modulare la risposta del sistema immune, bloccando la produzione di citochine Th1 e promuovendo una risposta immunitaria Th2, che solitamente è associata alla gravidanza normale. Inoltre, possiede un' attività immunoregolatoria sulle cellule B, di cui inibisce la proliferazione e la differenziazione, con conseguente riduzione della sintesi delle IgG. La valutazione dei livelli circolanti di vitamina $\mathrm{D}$ e la sua supplementazione, quando è insufficiente, può rappresentare un ausilio per incrementare le possibilità di portare a termine con successo la gravidanza. 